ARTICLE

\title{
Mechanism of phosphate sensing and signaling revealed by rice SPX1-PHR2 complex structure
}

Jia Zhou (10) 1,2,7, Qinli Hu (1) 1,2,7, Xinlong Xiao', Deqiang Yao ${ }^{3}$, Shenghong Ge ${ }^{1,2}$, Jin Ye (D) ${ }^{4}$, Haojie Li ${ }^{1}$, Rujie Cai ${ }^{5}$,

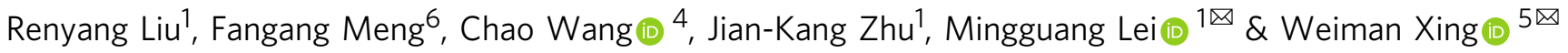

Phosphate, a key plant nutrient, is perceived through inositol polyphosphates (InsPs) by SPX domain-containing proteins. SPX1 an inhibit the PHR2 transcription factor to maintain Pi homeostasis. How SPX1 recognizes an InsP molecule and represses transcription activation by PHR2 remains unclear. Here we show that, upon binding InsP $\mathrm{P}_{6}, \mathrm{SPX} 1$ can disrupt PHR2 dimers and form a 1:1 SPX1-PHR2 complex. The complex structure reveals that SPX1 helix $\alpha 1$ can impose a steric hindrance when interacting with the PHR2 dimer. By stabilizing helix $\alpha 1$, Ins $\mathrm{P}_{6}$ allosterically decouples the PHR2 dimer and stabilizes the SPX1-PHR2 interaction. In doing so, InsP 6 further allows SPX1 to engage with the PHR2 MYB domain and sterically block its interaction with DNA. Taken together, our results suggest that, upon sensing the surrogate signals of phosphate, SPX1 inhibits PHR2 via a dual mechanism that attenuates dimerization and DNA binding activities of PHR2.

\footnotetext{
${ }^{1}$ Shanghai Center for Plant Stress Biology and Center of Excellence in Molecular Plant Sciences, Chinese Academy of Sciences, Shanghai 200032, China. 2 University of Chinese Academy of Sciences, Beijing 100049, China. ${ }^{3}$ State Key Laboratory of Oncogenes and Related Genes, Ren Ji Hospital, Shanghai Jiao Tong University School of Medicine, Shanghai 200127, China. ${ }^{4}$ MOE Key Laboratory for Membrane-less Organelles \& Cellular Dynamics, Hefei National Laboratory for Physical Sciences at the Microscale, School of Life Sciences, Division of Life Sciences and Medicine, University of Science and Technology of China, Hefei 230027, China. ${ }^{5}$ Shanghai Key Laboratory of Plant Molecular Sciences, College of Life Sciences, Shanghai Normal University, Shanghai 200234 , China. ${ }^{6}$ Beijing Neurosurgical Institute, Beijing Tiantan Hospital, Capital Medical University, Beijing 100070, China. ${ }^{7}$ These authors contributed equally:

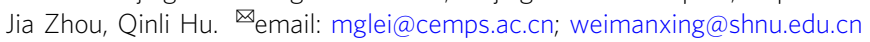


P hosphorus is a fundamental element of all living organisms and represents a key building block of many cellular molecules, such as the genetic material DNA and the energy carrier $\mathrm{ATP}^{1}$. Therefore, all cells need to maintain a sufficient concentration of phosphate $(\mathrm{Pi})$ in their cytoplasm ${ }^{2}$. For plants, the availability of inorganic $\mathrm{Pi}$ in soil is poor due to its low mobility ${ }^{3,4}$. To cope with the fluctuating levels of $\mathrm{Pi}$, plants have evolved sophisticated strategies to modulate $\mathrm{Pi}$ uptake and remobilization $^{5-9}$. The PHR transcription factors are the central regulators of $\mathrm{Pi}$ signaling, which bind to the PHR1-binding sequence (P1BS) in the promoter regions of phosphate starvation-induced (PSI) genes ${ }^{10}$. By activating the expression of PSI genes, PHRs enhance $\mathrm{Pi}$ uptake under Pi-deficient conditions $^{11,12}$. To avoid Pi toxicity caused by excessive $\mathrm{Pi}$ accumulation in plants, SPX proteins, on the other hand, bind to PHR proteins and inactivate PHR-induced transcription under Pi-replete conditions ${ }^{13,14}$.

How plants sense cellular Pi levels to manipulate PSI genes expression induced by PHR2 is a fascinating and key question in $\mathrm{Pi}$ homeostasis. The PHR proteins belong to the MYB-coiled-coil (MYB-CC) family, which is characterized by a conserved MYB DNA-binding domain and a potential CC dimerization domain ${ }^{11,15,16}$. In fact, dimerization of Arabidopsis PHR1 has been shown to be important for its high-affinity DNA binding function ${ }^{12}$. Previous studies indicated that rice SPX1 interacts with PHR2 in a Pidependent manner and acts as an inhibitor to repress PHR2 transcription activation under Pi-replete conditions ${ }^{17}$. These findings implied the important roles of SPX domain-containing proteins in sensing Pi. Recent structural analysis of the SPX-domain, in conjunction with in vivo studies, has provided compelling evidence supporting the functions of the SPX domain as Pi sensors. Interestingly, SPX domains do not appear to sense inorganic Pi directly, but instead recognize soluble inositol polyphosphates (InsPs), such as $\mathrm{InsP}_{7}$ or InsP $\mathrm{P}_{8}$, which act as a proxy for the cellular Pi status ${ }^{18,19}$. Such a mechanism has recently been unveiled by the crystal structures of SPX domains in complex with InsP $\mathrm{P}_{6}$, a commercially available substitute of $\operatorname{InsP}_{7}$ and $\operatorname{Ins}_{8}{ }^{18,20-22}$. Despite these recent advances, how SPX domain proteins recognize the InsP molecules and regulate downstream players in $\mathrm{Pi}$ homeostasis remains unclear. Here, we analyzed the effects of $\mathrm{InsP}_{6}$ binding to rice SPX1-PHR2 complex and determined the $\mathrm{InsP}_{6}$-bound SPX1-PHR2 complex structure. Together with biochemical studies, our structure unravels a unique dual mechanism by which SPX1 mediates Pi sensing and signaling.

\section{Results}

Ins $P_{6}$-induced SPX1-PHR2 heterodimer formation. Due to the instability of bacteria-produced full length PHR2 and SPX1 proteins, we purified an MYB and CC domain-containing PHR2 fragment (PHR2 $230-426$ ), which has been previously reported to interact with full length and a C-terminally truncated fragment of SPX1 (SPX1 $1^{1-259}$ ) (Supplementary Fig. 1a) ${ }^{17}$. To verify whether InsP molecules mediate SPX1-PHR2 complex formation, we used gel filtration assay to characterize the SPX1 $1^{1-259}-\mathrm{PHR} 2^{230-426}$ interaction. In contrast to no peak shift of PHR2 in absence of Ins $\mathrm{P}_{6}$, the peak of PHR2 ${ }^{230-426}$ proteins on the column shifted later and co-migrated with SPX1-259 proteins in presence of Ins $\mathrm{P}_{6}$, indicative of $\mathrm{Ins}_{6}$-induced specific interaction (Fig. 1a). Interestingly, the elution volume of PHR2 shifted from 12.2 to $13.2 \mathrm{ml}$ when forming a complex with SPX1, indicative of changes of the PHR2 dimer state. To verify whether PHR2 forms a dimer in solution as reported in the literature ${ }^{12}$, size exclusion chromatography with inline multi-angle light scattering (SEC-MALS) and crosslinking experiments were applied to measure the molecular weight of PHR2 $2^{230-426}$ and evaluate its oligomeric state. Isolated PHR2 $230-426$ appeared to exist in solution as a mixture of dimer and tetramer species based on both experiments (Fig. 1a and Supplementary Fig. 2a). Similar analysis of Arabidopsis PHR $1^{227-358}$, which contains the MYB and CC domains only, yielded a molecular weight of $29.2 \mathrm{kDa}$, indicative of a dimeric form (Supplementary Fig. 2b). We speculated that dimeric PHR2 was probably more prevalent in vivo and its tetrameric form might be detected in vitro due to nonspecific interactions. Based on the facts that SPX1-PHR2 complex was eluted later than PHR2 itself on the gel filtration column and that PHR2 might be a dimer in solution, whereas SPX1-259 is monomeric, we conclude that the SPX1 $1^{1-259}-\mathrm{PHR} 2^{230-426} \mathrm{com}$ plex most likely contains one copy of SPX1 $1^{1-259}$ and PHR2 $230-426$ each. Indeed, SEC-MALS analysis indicated that the $\operatorname{InsP}_{6^{-}}$ induced SPX1-PHR2 complex was eluted with a molecular mass of $50.1 \mathrm{kDa}$ (Fig. 1a). Hence, SPX1 and PHR2 formed a complex with a 1:1 stoichiometry in the presence of $\mathrm{InsP}_{6}$ and the PHR2 dimer was disrupted during the complex formation. It is noticed that SPX1 was slightly shifted by PHR2 as detected by size exclusion chromatography in the absence of $\operatorname{Ins}_{6}$ (Fig. 1a). However, it appeared to dynamically interact with the transcription factor but failed to form a stable and homogenous 1:1 heterodimer with PHR2.

If the stable binding of SPX1 to PHR2 hinges on PHR2 monomerization, a dimerization-defective mutant of PHR2 should be able to form a complex with SPX1 even in the absence of $\operatorname{InsP}_{6}$. Based on a recently reported Arabidopsis PHR1 CC dimer structure $^{20}$, we introduced several mutations that were expected to disrupt the PHR2 dimer interface. All PHR2 mutants, L348A, L358A, I362A, and L344R/Q351R/Q355R abolished the dimer formation in gel filtration and SEC-MALS assays (Fig. 1b). Strikingly, these PHR2 monomeric mutants, represented by the triple mutant (L348A/ L358A/I362A, PHR2 ${ }^{3 \mathrm{M}}$ ) could bind SPX1 with a Kd of $\sim 0.17 \mu \mathrm{M}$ in an $\mathrm{Ins}_{6}$-independent manner by gel filtration, SEC-MALS, and ITC assays (Fig. 1c, d). The binary complex was eluted from a gel filtration column at a similar position as the SPX1-PHR2 complex assembled by $\mathrm{InsP}_{6}$ and shared almost the same molecular weight of about $47.0 \mathrm{kDa}$.

Using ITC assay, we further analyzed the effect of Ins $_{6}$ on the DNA binding activity of PHR2. Individual $\mathrm{InsP}_{6}$ or SPX1 did not affect the ability of PHR2 to bind DNA. However, when both InsP $\mathrm{P}_{6}$ and SPX1 were mixed with PHR2, the DNA binding activity of the transcription factor was completely abolished (Fig. 1e). In line with the documented importance of PHR2 dimer ${ }^{12}$, we noticed that PHR2 monomeric mutant exhibited lower DNA binding and transcriptional activation capability compared with wild type in ITC and dualLUC transient transcriptional activity assays (Fig. 1e, f). In contrast to the wild type PHR2, the transcriptional activity of the PHR2 monomeric mutant could be inhibited by SPX1 under Pi starvation conditions in accordance with its constitutive interaction with SPX1. Based on these results and our aforementioned analysis shown in Fig. 1a, we conclude that PHR2 monomerization is a critical step in SPX1- and InsP $_{6}$-induced inactivation of the transcription factor.

Overall structure of SPX1-PHR2. The SPX1-PHR2 complex formation mediated by InsPs initiates downstream transcriptional inhibition of PSI genes. To understand how SPX1 responds to InsP binding, we determined the Ins $\mathrm{P}_{6}-\mathrm{SPX} 1-\mathrm{PHR} 2$ complex structure at $2.6 \AA$ resolution using single-wavelength anomalous diffraction. The model was refined to a final $R_{\text {work }}$ and $R_{\text {free }}$ value of 22.6 and $27.4 \%$, respectively (Supplementary Table 1 ). There are two amino acids located in the outlier region of Ramachandran plot probably due to poor accuracy associated with the electron density in the local region. The final model of the Ins $\mathrm{P}_{6-}$ SPX1-PHR2 complex contains two copies of the Ins $_{6}-\mathrm{SPX} 1-$ 
a

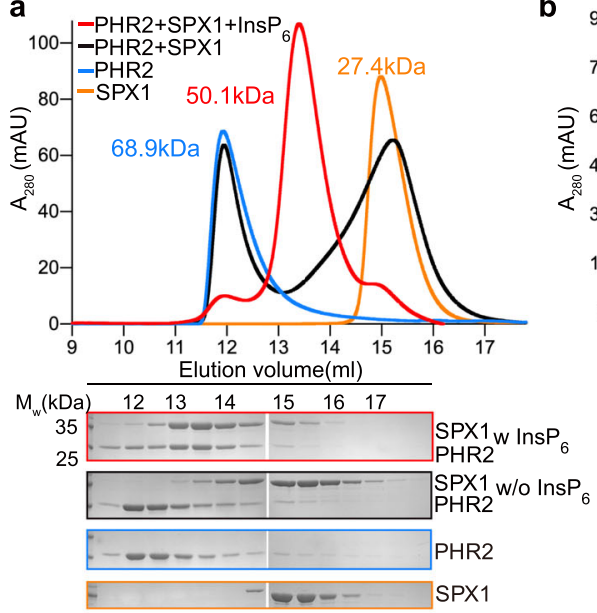

d

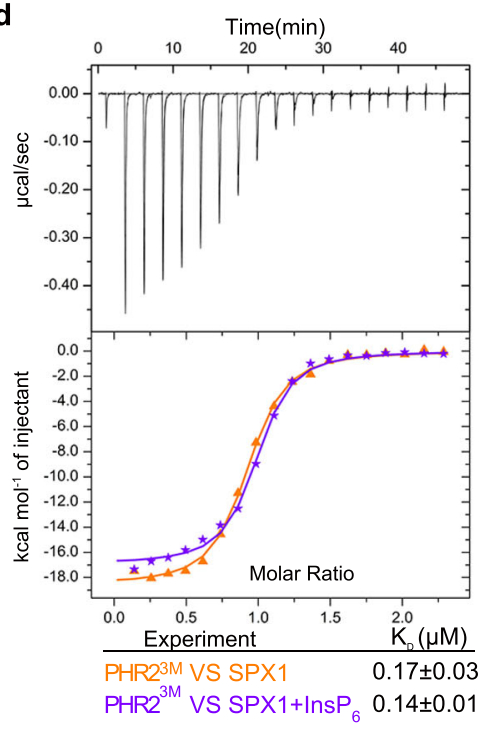

b

e

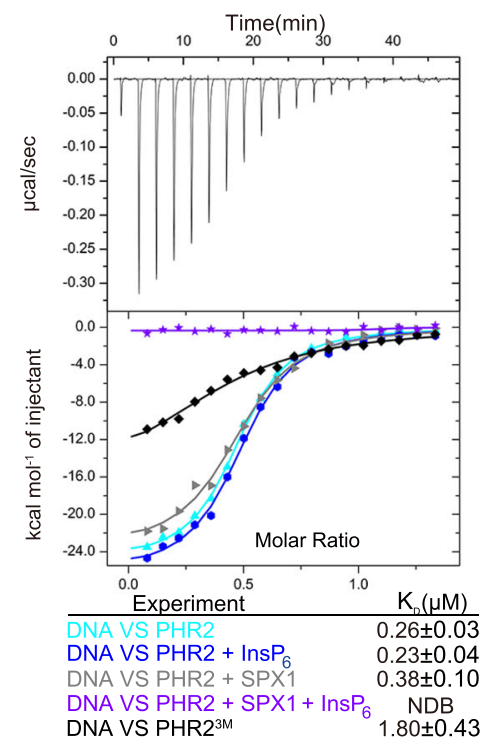

c

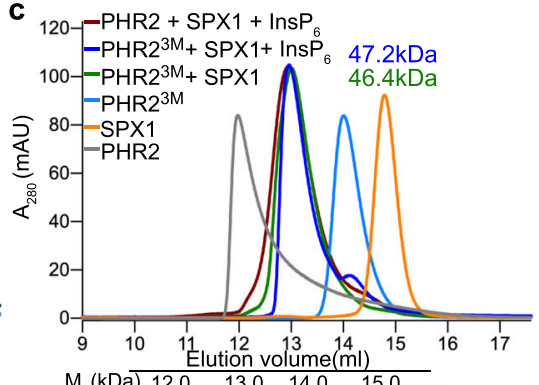

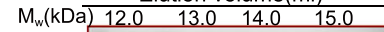
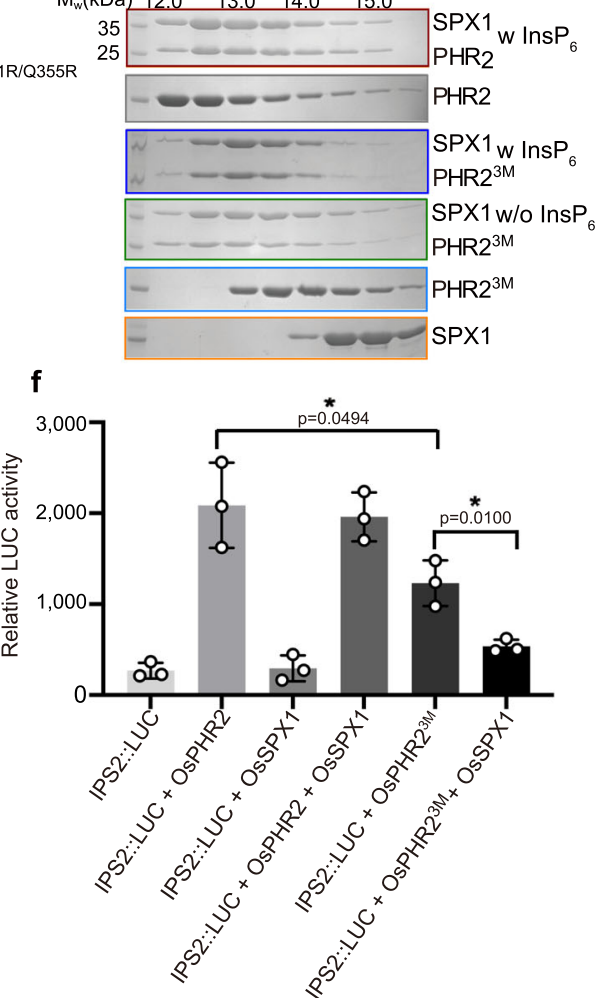

Fig. 1 Monomeric SPX1-PHR2 complex triggered by Ins $\mathbf{P}_{\mathbf{6}}$ was determinant for PHR2-DNA binding inhibition. a SPX11-259-PHR2230-426 complex formation was induced by InsP $_{6}$, as identified by gel filtration assay. (Upper) Gel filtration profiles of SPX11-259, PHR2 $230-426$, SPX1-259 and PHR2 $230-426$ in the presence or absence of $\operatorname{InsP}_{6}$ were color-coded. (Lower) Coomassie-blue stained SDS-PAGE gels of peak fractions. The molecular weights were labeled based on the SEC-MALS results. Experiments were independently repeated three times with similar results. Uncropped gel images are available as source data. b Mutations of PHR2 $230-426$ disassembled the PHR2 230-426 dimer into monomer. The molecular weights were measured by SEC-MALS.

Experiments were independently repeated three times with similar results. Uncropped gel images are available as source data. c Monomeric mutant of PHR2 230-426 L348A/L358A/I362A (PHR23M) could interact with SPX1'-259 in absence of InsP ${ }_{6}$. The molecular weights were measured by SEC-MALS.

Experiments were independently repeated three times with similar results. Uncropped gel images are available as source data. $\mathbf{d}$ Binding affinity of PHR2 $3 \mathrm{M}$ with SPX1 ${ }^{1-259}$ in the presence (purple) or absence of InsP $P_{6}$ (orange) were detected by ITC. e Effects of SPX1, InsP $\mathrm{P}_{6}$ and PHR2 ${ }^{3 \mathrm{M}}$ on PHR2-DNA(P1BS) binding affinity as detected by ITC. ITC curves for the titration of DNA (P1BS) with PHR2 230-426 (cyan); PHR2 230-426 and InsP 6 (blue); PHR2230-426 and SPX1-259 (gray); PHR2 $230-426$, SPX1 $^{1-259}$ and InsP ${ }_{6}$ (purple); PHR2 $3 \mathrm{M}$ (black). f Dimerization was important for the transcription activity of PHR2, and SPX1 can repress transcription activity of PHR2 $3 \mathrm{M}$ in phr1 phr2 mutant protoplast under Pi-deplete condition. Values are means \pm s.d. of three independent biological replicates. Sets of data were compared by paired-samples $T$ test (two-tailed), where the asterisk indicates statistically significant differences $\left({ }^{\star} P<0.05\right)$.

PHR2 complex in the asymmetric unit (Supplementary Fig. 1b). A highly crystallizable T4 lysozyme (T4L) was fused to the C-terminus of SPX1 to facilitate crystallization and improve the quality of crystals. The two T4 lysozyme molecules in the asymmetric unit adopt different orientations with respect to SPX1 due to crystal packing (Supplementary Fig. 1c).

The SPX domain of SPX1 consists of two long core helices $\alpha 3$ and a4, and two shorter C-terminal helices, $a 5$ and a6. Two N-terminal helices $\alpha 1$ and $\alpha 2$ form a helical hairpin. The two SPX1 molecules in the asymmetric unit, which share high structural homology with documented SPX domain structures, can be superimposed with each other with a Ca RMSD of $1.36 \AA$ (Supplementary Fig. 1d, e) ${ }^{18}$. The PHR2 MYB domain contains three consecutive $\alpha$ helices, which give rise to the conserved fold of the MYB proteins. The MYB domains of rice PHR2 and Arabidopsis PHR1 can be superimposed with a RMSD of $1.40 \AA$ (Supplementary Fig. 1f) ${ }^{23}$. The PHR2 CC motif long helix closely aligns with the CC motif of Arabidopsis PHR1 with a Ca RMSD of $0.89 \AA$ (Supplementary Fig. $1 g)^{20}$. Interestingly, both the MYB domain and CC motif of PHR2 make direct contact with SPX1. They approach SPX1 from two different directions and sandwich SPX1 in between with extensive protein-protein interfaces (Fig. 2a). 


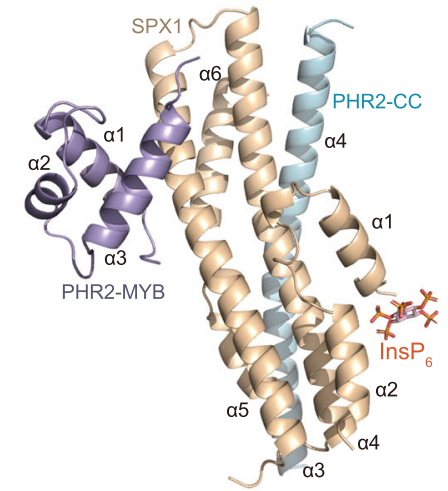

c

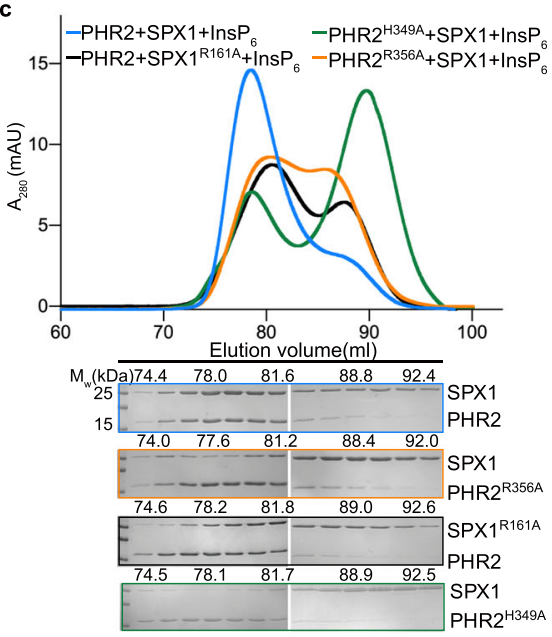

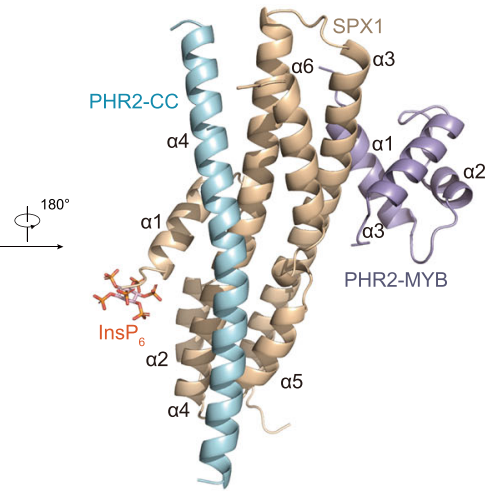

d

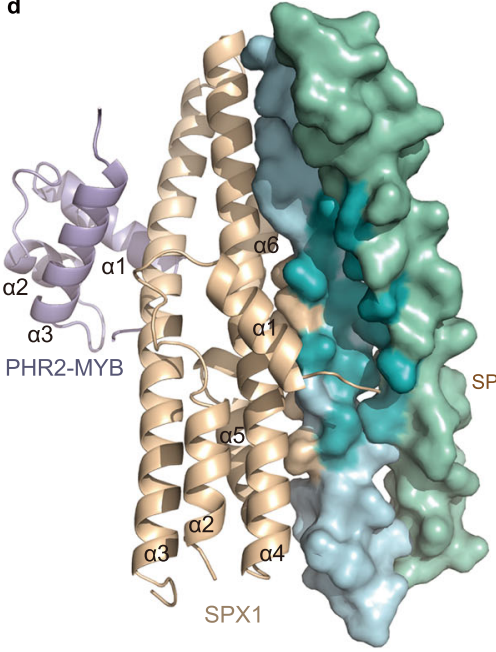

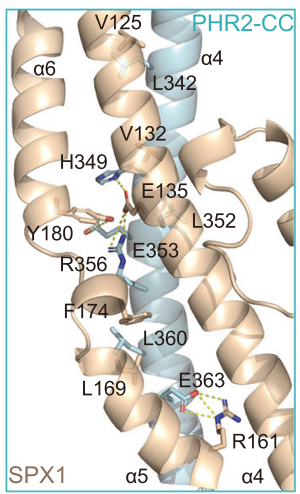

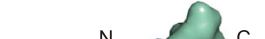

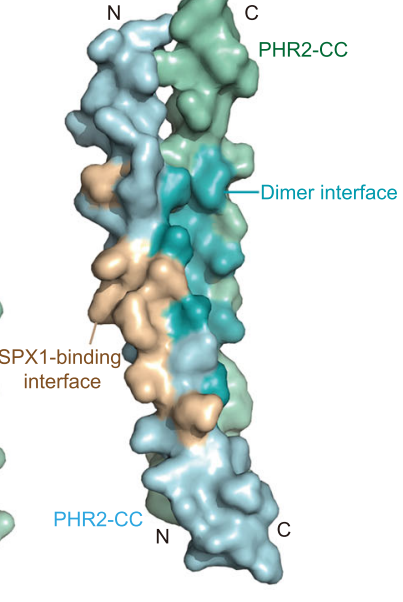

Fig. 2 SPX1-PHR2 CC interface is compatible with PHR2 dimer interface. a The crystal structure of InsP ${ }_{6}$-SPX1-PHR2 complex. SPX1 was colored in wheat, and the MYB and CC domains of PHR2 were colored in light blue and pale cyan respectively. Ins $\mathrm{P}_{6}$ was represented as a stick model in red. Ins $\mathrm{P}_{6}{ }^{-}$ SPX1-PHR2 complex was shown in two opposite views. The secondary structure elements and InsP 6 molecule were labeled. $\mathbf{b}$ Close-up view of SPX1-PHR2 CC domain interface. Critical interface residues were shown as sticks and labeled. The yellow dashed lines represent hydrogen bonds involved in the interaction of SPX1 and PHR2. c Mutations compromised the SPX1-198-PHR2 ${ }^{248-380}$ complex formation. (Upper) Gel filtration profiles of SPX1-198 and PHR2 248-380 were color-coded to show different SPX1'-198 mutants and PHR2 248-380 mutants. (Lower) Coomassie-blue stained SDS-PAGE gels of peak fractions. Experiments were independently repeated three times with similar results. Uncropped gel images are available as source data. d SPX1-PHR2 CC interface does not interfere with PHR2 dimerization. (Left) Superimposition InsP 6 -SPX1-PHR2-ternary complex structure onto AtPHR1 CC (PDB:6TO5) dimer structure. (Right) Surface representation of rice PHR2 CC dimer model. SPX1-binding interface was colored in wheat and PHR2 dimer interface was colored in teal.

SPX1-PHR2 CC motif interface. SPX1 interacts with the PHR2 CC motif via an extensive and elongated interface. Specifically, the PHR2 CC motif long helix interacts with three helices of SPX1 (a4, a5, and $\alpha 6)$ through several ionic interactions and hydrogen bonds (Fig. 2b). SPX1 E135 and R161 form salt-bridges with PHR2 R356 and E363 respectively. In addition, SPX1 E135, R161, and Y180 interact with PHR2 H349, E363, E353, and R356 through hydrogen bonds.

To verify the structural observations, we individually mutated residues in the SPX1-PHR2 CC motif interface into alanine. The R161A mutation in SPX1 and the PHR2 mutants, H349A and R356A, all compromised SPX1-PHR2 stable complex formation, whereas other SPX1 mutants, E135A and Y180A, and PHR2 mutants, E353A and E363A, failed to alter SPX1-PHR2 binding (Fig. $2 \mathrm{c}$ and Supplementary Fig. 3b, d). Moreover, hydrophobic residues, such as V125 and V132 in SPX1 and L352, L342 of PHR2 also contribute to the interface formation (Fig. 2b). Substitution of V125 and V132 in SPX1 and L342, L352 in PHR2 with alanine abolished SPX1-PHR2 interaction (Supplementary Fig. 3a, b). The L360 of PHR2 looks like participate the hydrophobic patch formation, however, PHR2 single mutation
L360A retained the ability to bind SPX1. Collectively, SPX1PHR2 CC interface, which is largely stabilized by hydrogen bonds, salt-bridges and hydrophobic interactions, is critical for complex formation.

The crystal structure of the Arabidopsis PHR1 CC dimer has recently been determined. Surprisingly, superposition analysis indicates that the PHR CC dimer interface is fully compatible with SPX1 binding (Fig. 2d), suggesting that the SPX1 binding via the SPX1-PHR2 CC interface itself does not interfere with PHR2 dimerization. In order to disrupt the PHR2 dimer, InsP $\mathrm{P}_{6}$ must act through a mechanism outside the SPX1-PHR2 CC interface.

Ins $\mathrm{P}_{6}$-binding site. Our structure reveals three $\mathrm{Ins}_{6}$ molecules in the asymmetric unit (Supplementary Fig. 4a). One InsP $\mathrm{P}_{6}$ molecule participates in crystal-packing interactions, while the other two are each captured by an SPX1 molecule via a highly basic surface groove formed between helices $\alpha 1, \alpha 2$, and a4 (Figs. 2a, 3a and Supplementary Fig. 4c). Importantly, this binding mode of these two Ins $\mathrm{P}_{6}$ molecules to SPX1 is very similar to the reported $\mathrm{InsP}_{6}$-SPX domain structures 

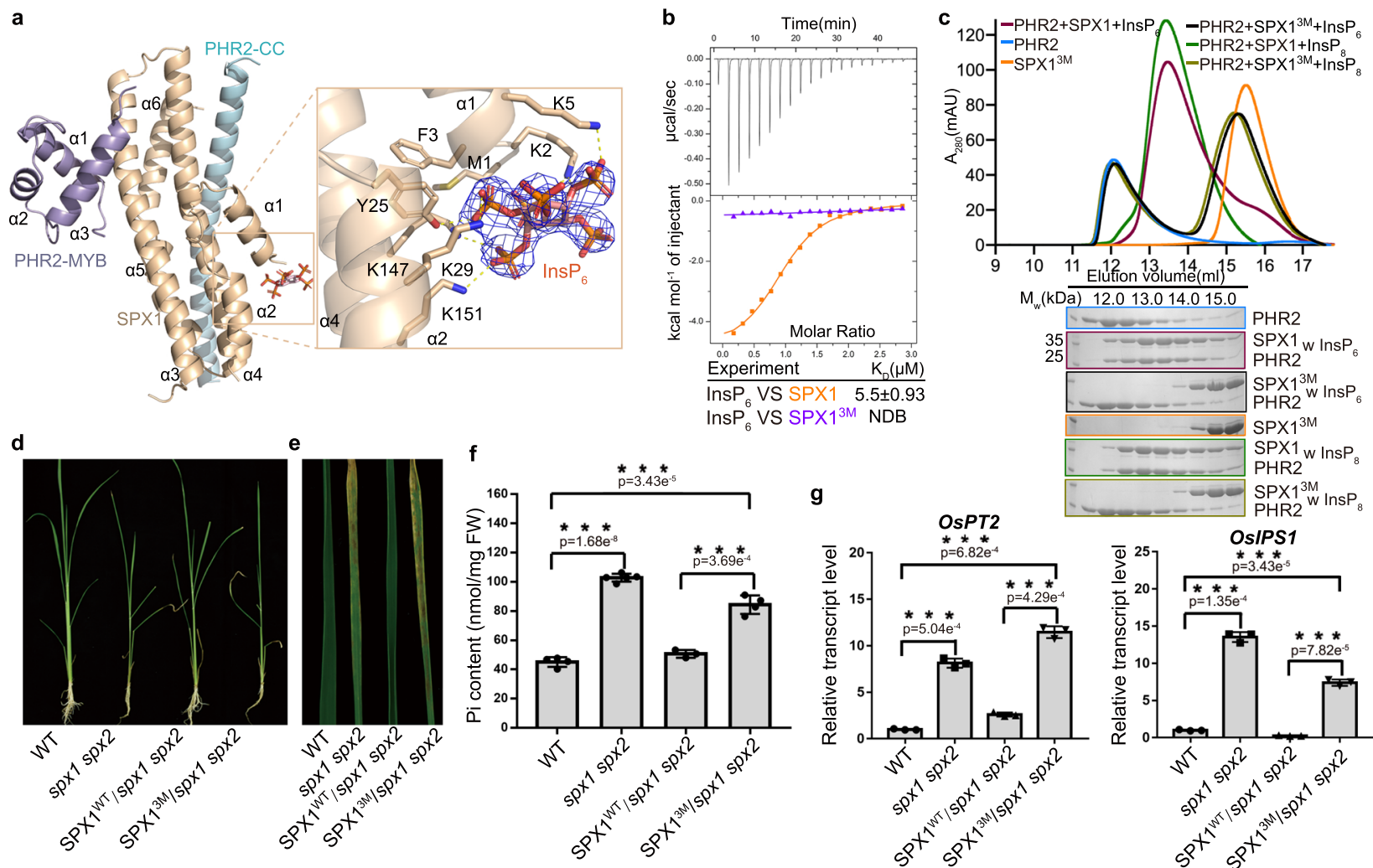
PHR2 SPX1 $1^{3 \mathrm{M}} \mathrm{W}_{\mathrm{PH} \mathrm{P}_{6}}$ SPX $1^{3 \mathrm{M}}$ $\mathrm{SPX} 1$
$\mathrm{SPX} 1$
$\mathrm{PHR}$ SPX1
PHR2 $\mathrm{SPX}_{1}^{3 \mathrm{M}} \mathrm{W}$ InsP

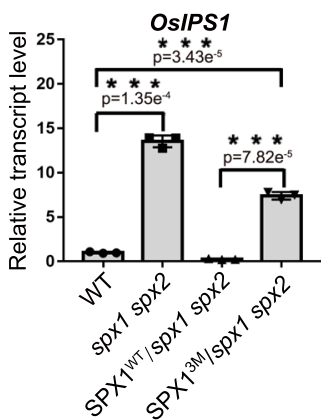

Fig. 3 Mutations in SPX1 Ins $\mathbf{P}_{\mathbf{6}}$-binding sites affect SPX1-PHR2 complex formation and $\mathbf{P i}$ signaling. a $A$ close-up view of the SPX1-InsP 6 interactions. Ins $\mathrm{P}_{6}$ was represented as a stick model with a stimulated annealing omit map and contoured at $1.1 \sigma$. Residues of SPX1 interacting with Ins $\mathrm{P}_{6}$ were shown as sticks and labeled. The yellow dashed lines represent hydrogen bonds involved in the interaction of $\mathrm{SPX}_{1}$ and InsP $\mathrm{P}_{6} \mathbf{b} \mathrm{Binding}$ affinities of InsP $\mathrm{P}_{6}$ with SPX1 $1^{1-259}$ (orange) and SPX1-259 Y25F/K29A/K151A (SPX1 ${ }^{3 \mathrm{M}}$, purple) were measured by ITC. NDB, no detectable binding. c Mutations of InsP 6 -binding sites in SPX11-259 impaired the SPX1-259-PHR2 230-426 interaction. The assays were performed as described in (Fig. 1a). Experiments were independently repeated three times with similar results. Uncropped gel images are available as source data. $\mathbf{d}$ Growth phenotype of WT, spx 1 spx2, and two kinds of transgenic plants were grown under Pi-sufficient condition (SPX1WT/spx1 spx2 and SPX1Y25F/K29A/K151A/spx1 spx2). e Phenotype of phosphate excess in leaves of plants shown in (d). $\mathbf{f}$ Pi contents of plants shoots shown in (d). Error bars, mean \pm s.d. The number of biologically independent samples of WT, spx1 spx2, SPX1WT/spx1 spx2 and SPX13M/spx1 spx2 is 4, 5, 3 and 4, respectively. Sets of data were analyzed by independent-samples T test (two-tailed), where ${ }^{\star \star \star}$ represents a statistically significant difference at $p<0.001$. $\mathbf{g}$ PSI genes (OsIPS1 and OsPT2) expression in the root of the plants shown in (d). Error bars, mean \pm s.d. The bar graphs show the results from three biologically independent samples $(n=3$ ). Sets of data were compared by pairedsamples T-test (two-tailed), where ${ }^{\star \star \star}$ represents a statistically significant difference at $p<0.001$.

(Supplementary Fig. 4b). Three lysine residues (K29, K147, and K151) and a tyrosine residue (Y25) from SPX1 contribute to the interaction with $\mathrm{InsP}_{6}$ (Fig. 3a and Supplementary Fig. 5). Their functional importance is underscored by their conservation among SPX domains from different eukaryotes (Supplementary Fig. 6a). To validate the roles of these conserved amino acids in Ins $_{6}$ binding, a triple mutation of SPX1, Y25F/K29A/K151A, was designed and tested in an ITC assay. In contrast to the moderate Ins $\mathrm{P}_{6}$ binding ability of the wild-type protein, the SPX1 mutant lost its Ins $\mathrm{P}_{6}$-binding activity, confirming the importance of these three residues for $\mathrm{InsP}_{6}$ perception (Fig. 3b). SPX1 $\mathrm{K} 147 \mathrm{~A}$ mutation had little effect on $\mathrm{Ins}_{6}$ binding affinity (Supplementary Fig. 7).

Consistent with the notion that $\mathrm{InsP}_{6}$ mediates the disruption of the PHR2 dimer outside the SPX1-PHR2 CC interface, our structure shows that the SPX1 InsP $\mathrm{P}_{6}$-binding site is removed from the long PHR2 CC helix. The closest residue of PHR2, Q359, is about $13 \AA$ away from Ins $\mathrm{P}_{6}$, which nevertheless is required for the stable SPX1-PHR2 interaction as shown in our size exclusion chromatography analysis. To further confirm the critical role of InsP molecules in stabilizing the 1:1 SPX1-PHR2 heterodimeric complex, we measured the binding of the SPX1 Y25F/K29A/ K151A triple mutant with PHR2 using gel filtration assay. As shown in Fig. 3c, the SPX1 triple mutant could not co-migrate with PHR2 despite the presence of Ins $\mathrm{P}_{6}$ or Ins $\mathrm{P}_{8}$, indicative of weak or no binding between SPX1 triple mutation and PHR2. Importantly, rice SPX1 Y25F/K29A/K151A triple mutant could not rescue $s p \times 1 s p \times 2$ double mutant phenotype (Fig. $3 \mathrm{~d}$, e). Not only the Pi content in the triple mutant was increased compared with wild type, PSI genes were also up-regulated (Fig. $3 \mathrm{f}, \mathrm{g}$ ). These data support the idea that the binding of $\operatorname{Ins}_{6}$ at this allosteric site somehow enables SPX1 to interact with and inhibit PHR2.

In the crystal, we notice that the SPX1 helix a 1 residues $\mathrm{M} 1$, $\mathrm{K} 2, \mathrm{~F} 3$, and $\mathrm{K} 5$ also participate in interacting with $\operatorname{InsP}_{6}$ (Fig. 3a and Supplementary Fig. 5). Deletion of the N-terminal helix a1 $(\triangle \mathrm{N} 17)$ reduced $\mathrm{InsP}_{6}$ binding affinity by $\sim 6$-fold (Fig. $4 \mathrm{a}$ ). But this N-terminally truncated SPX1 mutant still retained the ability to bind Ins $_{6}$. These results suggest that $\mathrm{N}$-terminal helix $\alpha 1$ is not the major player in $\operatorname{Ins}_{6}$ binding. Nonetheless, the four Ins $\mathrm{P}_{6}$-contacting residues in the $\alpha 1$ helix of SPX 1 are also strictly conserved in SPX1 orthologs (Supplementary Fig. 6a), hinting at an important functional role that is coupled to $\mathrm{Ins}_{6}$ binding.

Mechanism of action of InsP $\mathbf{P}_{\mathbf{6}}$. To further dissect the mechanism by which $\mathrm{InsP}_{6}$ helps disrupt the PHR2 dimer, we superimposed 

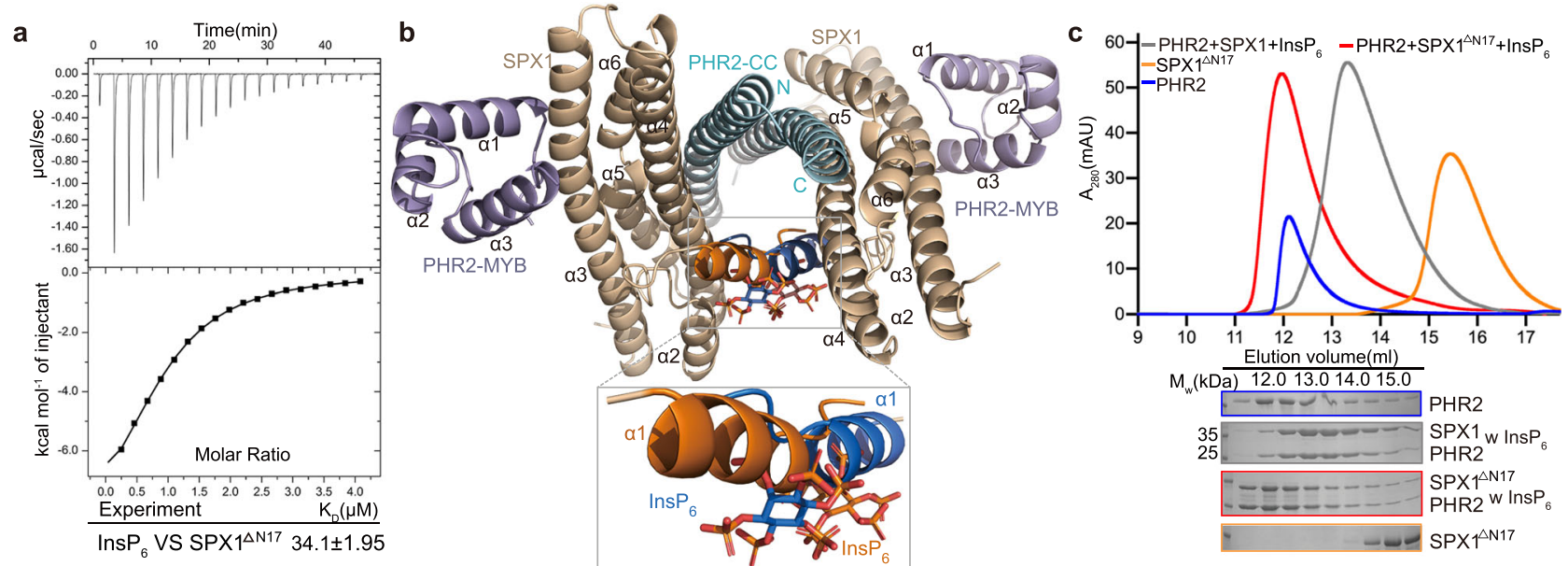

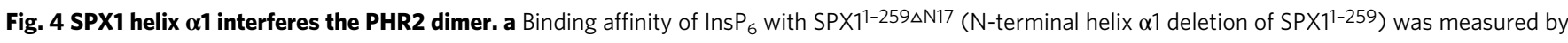
ITC. b PHR2 dimer model constructed by superimposing PHR2 CC in InsP $\mathrm{F}_{6}$-SPX1-PHR2 ternary complex structure with AtPHR1 CC dimer structure. The obvious clash occurred between the two helixes $\alpha 1$ was shown in the close-up view. Two helixes $\alpha 1$ of SPX1 were represented in orange and blue respectively. c SPX1-259 $\Delta$ N17 impaired the SPX1-259-PHR2 230-426 interaction. SPX1 helix $\alpha 1$ is essential for PHR2 dimer dissociation. The assays were performed as described in (Fig.1a). Experiments were independently repeated three times with similar results. Uncropped gel images are available as source data.

the PHR2 CC motif of our InsP $\mathrm{P}_{6}$-SPX1-PHR2 ternary complex structure onto the Arabidopsis PHR1 CC dimer structure. Remarkably, the resulting model readily revealed a severe collision between the $a 1$ helices of the two SPX1 molecules (Fig. 4b). This observation strongly suggests that $\operatorname{InsP}_{6}$ stabilizes SPX1 a1 helix and reinforces a steric hindrance to prevents the SPX1bound PHR2 to maintain its dimeric state. To validate this idea, we tested the ability of the SPX1 mutant missing its N-terminal a1 helix to disrupt the PHR2 dimer in the presence of InsP $_{6}$. Although this SPX1 mutant retains its $\mathrm{InsP}_{6}$-binding activity as shown in Fig. 4a, it indeed failed to separate the PHR2 dimer (Fig. 4c and Supplementary Fig. 8). To visualize the effect of Ins $\mathrm{P}_{6}$ on the SPX1 a1 helix, we obtained Small Angle X-ray Scattering (SAXS) data for SPX1 and InsP $\mathrm{P}_{6}$-SPX1. The comparison of scattering curves between SPX1 and InsP ${ }_{6}$-bound SPX1 shows that $\mathrm{InsP}_{6}$-bound state appears to be different. It seems that InsP makes SPX1 pack tightly according to the smaller Guinier radius of gyration ( $\mathrm{Rg}$ ) and Dmax for SPX1 in presence of $\mathrm{InsP}_{6}$ (Supplementary Fig. 9 and Supplementary Table 2). Further, we calculated the respective molecular bead model using SAXS profiles, and reconstructed the SPX1 model by SASREF. SPX1 and InsP $\mathrm{P}_{6}$-bound SPX1 show similar architecture except $\alpha 1$ helix. In addition, the $\mathrm{Ab}$ initio molecular envelope shows a difference around al helix area (Supplementary Fig. 10). Further, the thermal shift assay showed that Ins $\mathrm{P}_{6}$ could increase the melting temperature (Tm) of SPX1 greatly but could not change the Tm of SPX1 $1-259 \triangle \mathrm{N} 17$ obviously (Supplementary Fig. 11c, d). The comparison of Circular Dichroism (CD) spectra of SPX1 and Ins $\mathrm{P}_{6}$-SPX1 indicated that Ins $\mathrm{P}_{6}$ could increase the helical content of SPX1 (Supplementary Fig. 11a, b). Therefore, InsP ${ }_{6}$ could stabilize SPX1 al helix. Notably, the corresponding helix al truncation mutation in PHO1, an SPX1 domain-containing protein, exhibited a similar phenotype as $\mathrm{SPX} 1 \mathrm{Ins}_{6}$-binding site triple mutation ${ }^{18}$. Collectively, the location of the $\operatorname{Ins}_{6}$-binding site distant from the SPX1-PHR2 CC interface, the involvement of the SPX1 a1 helix in $\mathrm{InsP}_{6}$ binding, and the steric hindrance imposed by SPX1 a1 helix in the context of the PHR2 dimer, provide a structural mechanism by which $\mathrm{Ins}_{6}$ allosterically uncouples the PHR2 dimer and promotes the formation of an SPX1-PHR2 1:1 heterodimeric complex.
Interface between SPX1 and PHR2-MYB. In addition to breaking the PHR2 dimer apart, our structure shows that SPX1 forms a second interface with PHR2 via its MYB domain. The helices $\alpha 1$ and $\alpha 3$ of the PHR2 MYB domain act as a clamp to hold the helix $a 3$ of SPX1 through a salt-bridge and side-chain hydrogen bond network, which is formed among E89, E92, K99, E100 of SPX1 and K297, H294, R248, E257, R302 of PHR2 (Fig. 5a). To verify the structure, we individually mutated residues in the SPX1-PHR2 MYB interface into alanine. SPX1 mutants E89A, K99A, E92A, and E100A impaired the interaction with PHR2 and PHR2 mutants K297A, H294A, R302A attenuated the binding with SPX1. Other mutants PHR2 R248 and E257 had little effect on the SPX1-PHR2 interaction. These results suggest that this second SPX1-PHR2 interface is also important for SPX1-PHR2 complex assembly (Fig. 5b and Supplementary Fig. 3c, d).

Remarkably, superposition analysis of our structure and the Arabidopsis PHR1 MYB-DNA complex structure reveals a severe steric hindrance for DNA binding by the SPX1-bound PHR2MYB. In fact, the PHR2 MYB domain contacts with SPX1 through the same helix $\alpha 3$ and Loop 1 region, which is used by Arabidopsis PHR1 to dock onto DNA (Fig. 5c). This feature of the Ins $\mathrm{P}_{6}$-stabilized SPX1-PHR2 complex provides the structural basis explaining the previous observation that SPX1 competes with DNA for PHR2 MYB binding in vitro and ultimately inhibits the transcriptional activation of PHR2-induced PSI genes. The functional importance of this SPX1-PHR2 MYB interface is further underscored by the conservation of the majority of the interface residues in plants (Supplementary Fig. 6a, b). In fact, Arabidopsis PHR1 mutants K325A/R335A and $\mathrm{K} 325 \mathrm{~A} / \mathrm{H} 328 \mathrm{~A} / \mathrm{R} 335 \mathrm{~A}$, which have residues at the predicted SPX1-binding interface (R335 and $\mathrm{H} 328$ are the PHR1 residues corresponding to rice PHR2 R356 and H349), showed a Pi excess phenotype ${ }^{20}$.

\section{Discussion}

Our InsP $\mathrm{P}_{6}$-SPX1-PHR2 structure predicates the incompatibility of PHR2 homodimerization and SPX1 binding due to the collision of two SPX1 helices al in superimposition analysis and mechanistically explains the ability of SPX1 in disrupting PHR2 

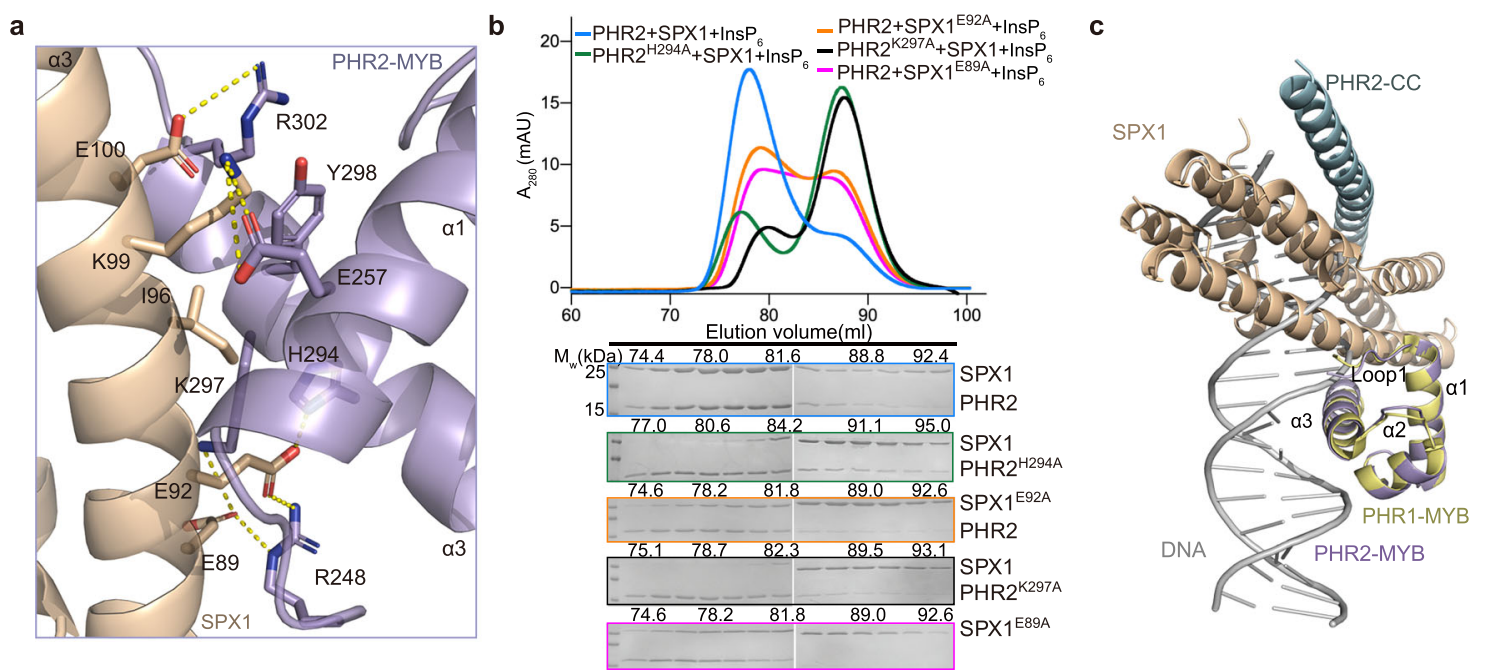

Fig. 5 Structural basis for PHR2 DNA binding inhibition by SPX1. a Close-up view of SPX1-PHR2 MYB interface. Critical interface residues were shown as sticks and labeled. The yellow dashed lines represent hydrogen bonds. b Mutations disrupted the SPX1-198_PHR2 ${ }^{248-380}$ complex formation. (Upper) Gel filtration profiles of SPX1-198 mutants and PHR2 248-380 mutants were color-coded. (Lower) Coomassie-blue stained SDS-PAGE gels of peak fractions. Experiments were independently repeated three times with similar results. Uncropped gel images are available as source data. c SPX1 occupied the PHR2 MYB-DNA binding region. Helix $\alpha 3$ and loop 1 of MYB involve in DNA binding and SPX1 interacting based on superimposition of SPX1-PHR2 complex and AtPHR1 MYB-DNA complex (PDB:6J4R).

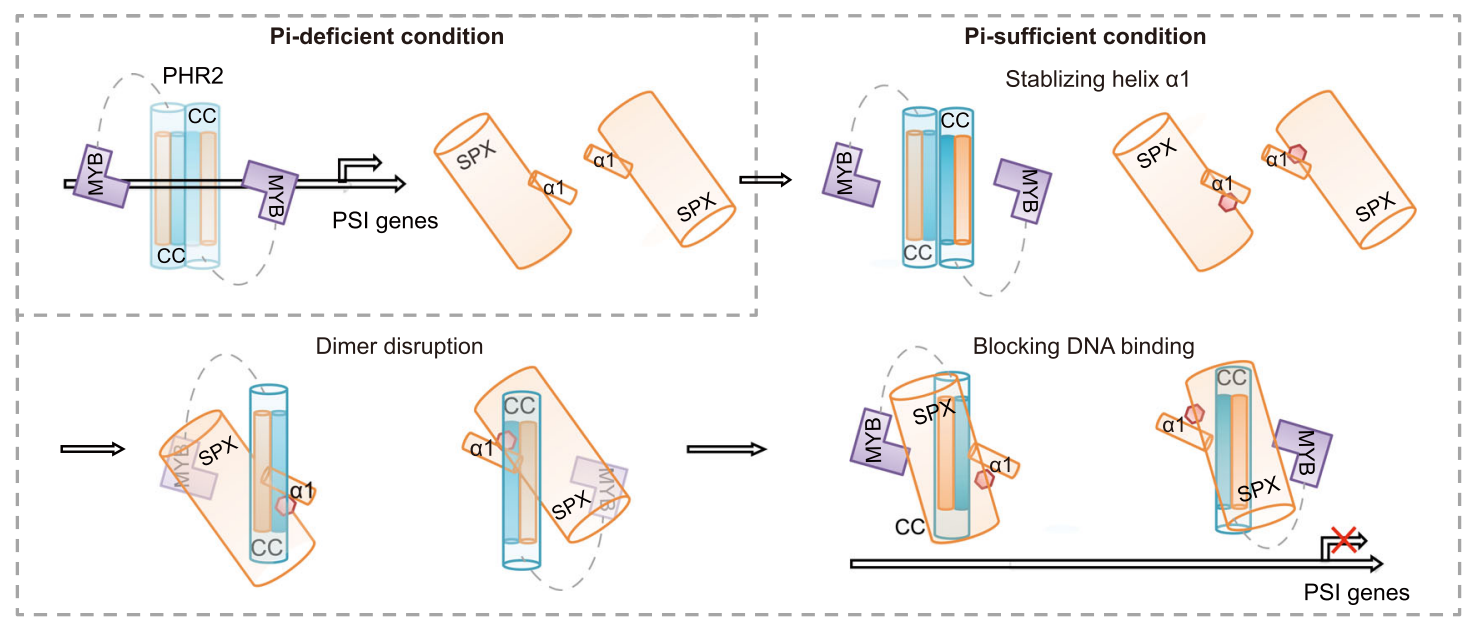

Fig. 6 InsP $\mathbf{6}_{\mathbf{6}}$-induced and SPX1-mediated PHR2 dimer dissociation and DNA binding inhibition. A proposed model for InsP molecule induced recognition and inhibition of PHR2 by SPX1. Under Pi-deficient condition, PHR2 existed as a dimer to bind to the P1BS, and activated the transcription of PSI genes. Under Pi-sufficient condition, InsP molecule could be recognized by SPX1 and stabilized the helix $\alpha 1$ of SPX1 with InsP molecule concentration increasing. Then, PHR2 dimer was disrupted, allowing SPX1 interacted with MYB and CC domains of PHR2 simultaneously. Thus, the transcriptional activation of PSI genes was repressed. The red octagon in the model represented InsP molecule.

dimer in the presence of InsP. Interestingly, the $\mathrm{InsP}_{6}$ binding site is not situated at the SPX1-PHR2 interface. But the loss of $\mathrm{Ins}_{6}$ binding ability clearly affects the stable interaction between SPX1 and PHR2. Based on these observations, we conclude that InsP $_{6}$ plays an important role in SPX1-mediated PHR2 dimer dissociation. A similar effect on PHR2 attenuation was observed in SPX1 helix $\alpha 1$, whose deletion did not affect InsP $\mathrm{P}_{6}$ binding greatly, but prevented the dimer separation despite of helix al absence on the SPX1-PHR2 interaction interface. However, neither InsP ${ }_{6}$ nor SPX1 itself could abolish the PHR2 dimer (Fig. 1a and Supplementary Fig. 12a, b). Since helix al stabilizes SPX1Ins $_{6}$ interaction, Ins $\mathrm{P}_{6}$ is expected to elicit the same effect on SPX1 helix a1, which leads to the destabilization of the PHR2 dimer. We hypothesize that the InsP molecule is sensed by SPX1 and binds to SPX1 with the increase of InsP concentration. Each
InsP molecule from two SPX1 molecules probably stabilizes SPX1 helix $\alpha 1$ to disrupt PHR2 dimer. Concurrently with the dimer dissociation, PHR2 MYB domain is occupied by SPX1, and its DNA binding is blocked and hence loses the ability to promote expression of PSI genes (Fig. 6a).

Many signal molecules transmit signal to downstream through a common allosteric mechanism such as gibberellin and strigolactone ${ }^{24,25}$. InsP molecules allosterically triggered PHR2 dimer separation, a key step for complex formation, which is reminiscent of Abscisic acid (ABA). ABA could weaken ABA receptor PYL dimer, and monomeric state of $A B A$ receptor such as PYL10 and PYL13 could mediate ABA-independent inhibition of PP2C ${ }^{26,27}$. This is in line with our result that monomeric PHR2 could directly interact with SPX1 independently of InsP molecule. $\mathrm{ABA}$ induces conformational changes on the dimer stabilization 
CL2 loop of PYL1 and weakens the dimer interface ${ }^{28}$. However, our InsP ${ }_{6}$-SPX1-PHR2 ternary complex structure and assays indicate that $\mathrm{InsP}_{6}$ may attenuate PHR2 dimer by stabilizing SPX1 helix $\alpha 1$, which is different from the conformational changes induced by ABA. By deciphering the InsP sensing and signaling mechanism of SPX1, our findings shed light on a key process of plant adaptations to variations in nutrient availability and pave the road for engineering phosphate efficiency in crops.

\section{Methods}

Protein expression and purification. The truncations and mutants of SPX1 or PHR2 were cloned into the modified pET21a vector (Novagen) providing a tobacco etch virus (TEV) cleavable N-terminal 10xHis-MsyB tag. Sequences for primers are listed in Supplementary Table 3. The proteins were overexpressed in E. coli BL21 (DE3). Cells were grown to $\mathrm{OD}_{600 \mathrm{~nm}}$ of 0.6 at $37^{\circ} \mathrm{C}$, then reduced to $16^{\circ} \mathrm{C}$ and induced with $0.1 \mathrm{mM}$ isopropyl $\beta$-D-1-thiogalactopyranoside for $8-10 \mathrm{~h}$. Cells were harvested by centrifugation for $15 \mathrm{~min}$ at $5300 \times \mathrm{g}$ and resuspended in buffer $\mathbf{A}$ (50 mM Tris/ $\mathrm{HCl} \mathrm{pH} \mathrm{8.0,} 200 \mathrm{mM} \mathrm{NaCl}, 20 \mathrm{mM}$ imidazole, $1 \mathrm{mM}$ TCEP). Lysates were cleared by centrifugation at $37,000 \times g$ for $1 \mathrm{~h}$. The supernatant was loaded onto a $\mathrm{Ni}^{2+}$ affinity column (GE Healthcare). Then, proteins were eluted with buffer A supplemented with $250 \mathrm{mM}$ imidazole. The proteins were loaded onto an anion-exchange column (Source-15Q, GE Healthcare). The target proteins were separated by increasing the salt concentration from buffer $\mathbf{B}(20 \mathrm{mM}$ Tris/ $\mathrm{HCl}$ pH $8.0,100 \mathrm{mM} \mathrm{NaCl}, 1 \mathrm{mM}$ TCEP) to the same buffer containing $1 \mathrm{M} \mathrm{NaCl}$ through a linear gradient. After cleavage by TEV protease at $4{ }^{\circ} \mathrm{C}$ for $12 \mathrm{~h}$, the proteins were purified by heparin column with the same buffer used in anion-column purification. The target proteins were further purified by Size Exclusion Chromatography (SEC, Superdex 200 Increase 10/300 GL, GE Healthcare) with the buffer C containing $20 \mathrm{mM}$ Tris/HCl pH 8.0, $200 \mathrm{mM} \mathrm{NaCl}, 1 \mathrm{mM}$ TCEP. The complex of SPX1 and PHR2 were assembled by mixing individually purified equimolar SPX1 and PHR2 with Ins $\mathrm{P}_{6}$ (Sigma, catalog \#P8810) and incubating at $4{ }^{\circ} \mathrm{C}$ for $12 \mathrm{~h}$, then purified by SEC with the buffer $\mathbf{D}$ containing $200 \mathrm{mM} \mathrm{NH}_{4} \mathrm{HCO}_{3} \mathrm{pH} 8.6,1 \mathrm{mM}$ TCEP for crystal screening.

Crystallization, data collection, and structure determination. The crystals of PHR2 (residues 248-380) and SPX1 (residues 1-198) complex showed obvious anisotropy, and the T4-lysozyme was fused to SPX1 for improving the crystal quality. The complex crystals were grown at $4{ }^{\circ} \mathrm{C}$ by the hanging-drop vapor diffusion method, using $1.5 \mu \mathrm{l}$ protein complex sample mixed with an equal volume of reservoir solution $(0.1 \mathrm{mM}$ sodium phosphate dibasic/citric acid, $\mathrm{pH} 4.2,200 \mathrm{mM}$ $\mathrm{NH}_{4} \mathrm{HCO}_{3}, 10 \%(\mathrm{w} / \mathrm{v})$ PEG3350). Furthermore, good quality crystals were subjected to a post-crystallization dehydration procedure by gradually increasing the concentration of PEG3350 to $20 \%$, then directly frozen in liquid nitrogen with cryoprotectant $(0.1 \mathrm{mM}$ sodium phosphate dibasic/citric acid pH 4.2, $200 \mathrm{mM}$ $\mathrm{NH}_{4} \mathrm{HCO}_{3}, 18 \%$ (w/v) PEG3350, 20\% glycerol). These procedures improved the resolution of the crystal to $2.6 \AA$. PHR2 $248-380 \mathrm{~V} 263 \mathrm{M} / \mathrm{L} 278 \mathrm{M} / \mathrm{L} 295 \mathrm{M} / \mathrm{L} 340 \mathrm{M}$ mutation, with no influence on SPX1 binding, was used to facilitate structure determination. The phase of the SPX1 ${ }^{1-198-T 4}$ lysozyme-Se-PHR2 248-380 V263M/L278M/L295M/L340M complex was calculated by single-wavelength anomalous dispersion method using data collected at the peak wavelength of selenium $(\lambda=0.979 \AA)$. All data sets were collected at beamlines BL17U1 and BL19U1 at the Shanghai Synchrotron Radiation Facility. The crystals belonged to space group $\mathrm{P} 2{ }_{1} 2_{1} 2_{1}$ with unit cell parameters $a=65.24 \AA, b=107.49 \AA, c=174.52 \AA$, and contained two SPX1-PHR2 complex molecules in an asymmetric unit. Reflection data were indexed, integrated, and scaled with $\mathrm{XDS}^{29}$. The structural model was manually built, refined, and rebuilt using COOT and PHENIX ${ }^{30,31}$. The final refinement statistics are summarized in Supplementary Table 1 .

Gel filtration assay. The HiLoad $16 / 600$ Superdex 200 pg column and Superdex 200 Increase 10/300 GL column were used for the SEC analysis. The assay was performed with an injection volume of $1 \mathrm{~mL}$, and the buffer $\mathrm{C}(20 \mathrm{mM}$ Tris/ $\mathrm{HCl}$ pH 8.0, $200 \mathrm{mM} \mathrm{NaCl}, 1 \mathrm{mM}$ TCEP) with or without InsP ${ }_{6}$ (Figs. 1b, c, 2c, 4c, 5b, and Supplementary Figs. 3a-d, 12a, b), the buffer $\mathrm{C}$ or with $\mathrm{InsP}_{6} / \mathrm{InsP}_{8}$ (Fig. 3c), and buffer $\mathbf{E}\left(200 \mathrm{mM}\right.$ PBS pH7.4) or with InsP $_{6}$ (Fig. 1a). All the samples were incubated at $4{ }^{\circ} \mathrm{C}$ for $12 \mathrm{~h}$. The peak fractions were analyzed by SDS-PAGE followed Coomassie Brilliant Blue staining.

SEC coupled with multi-angle light scattering. The analysis was performed on an AKTA Pure system (GE Healthcare) coupled with a static light scattering detector (miniDawn, Wyatt) and a differential refractive index detector (Optilab, Wyatt). Protein samples (concentration of 2-3 $\mathrm{mg} \mathrm{ml}^{-1}$ ) were filtered and loaded onto a Superdex 200 Increase 10/300 GL column pre-equilibrated by the buffer $\mathbf{C}$ (20 mM Tris/ $\mathrm{HCl} \mathrm{pH} 8.0,200 \mathrm{mM} \mathrm{NaCl}, 1 \mathrm{mM}$ TCEP) with or without $1 \mathrm{mM}$ Ins $_{6}$. Data were analyzed and drawn with ASTRA7 (Wyatt). The experiments were performed at $4{ }^{\circ} \mathrm{C}$.
Isothermal titration calorimetry. The Isothermal Titration Calorimetry (ITC) experiments were carried out on a Microcal PEAQ-ITC instrument (Malvern). All titrations were performed at $25^{\circ} \mathrm{C}$. Samples in cell and syringe were both dialyzed against buffer E (200 mM PBS pH7.4) (Fig. 1e) or buffer F ( $20 \mathrm{mM}$ HEPES/NaOH pH 7.5, $200 \mathrm{mM} \mathrm{NaCl}, 2 \mathrm{mM} \beta$-mercaptoethanol) (Figs. 1d, 3b, 4a and Supplementary Fig. 7) prior to all titrations. To measure the binding affinity between P1BS (CGCG AATATTCCCA) and PHR2 ${ }^{230-426}, \mathrm{PHR}^{230-426}-\mathrm{SPX}^{1-259}, \mathrm{PHR}^{230-426}-\mathrm{InsP}_{6}$, PHR2 ${ }^{230-426}$-SPX1 $^{1-259}-$ InsP $_{6}$ or PHR2 ${ }^{230-426 ~ L 348 A / L 358 A / I 362 A ~}, 40 \mu \mathrm{l} \mathrm{P1BS}$ at $70 \mu \mathrm{M}$ was injected into $280 \mu \mathrm{L}$ protein samples at $10 \mu \mathrm{M}$ respectively. To measure the binding affinity between SPX1 $1^{1-259}, \mathrm{SPX}^{1-259} \mathrm{Y} 25 \mathrm{~F} / \mathrm{K} 29 \mathrm{~A} / \mathrm{K} 151 \mathrm{~A}$ or SPX1 $1^{1-259} \mathrm{~K} 147 \mathrm{~A}$ and InsP $_{6}, 40 \mu \mathrm{InsP}_{6}$ at $600 \mu \mathrm{M}$ in the syringe was injected into $280 \mu \mathrm{L}$ SPX1 ${ }^{1-259}$, SPX1 $1^{1-259 Y 25 F / K 29 A / K 151 A}$ and SPX1 ${ }^{1-259 K 147 A}$ at $40 \mu \mathrm{M}$ in the cell, respectively. To measure the binding affinity between $\mathrm{SPX}^{1-259 \Delta \mathrm{N} 17}$ and $\mathrm{InsP}_{6}, 40 \mu \mathrm{lnsP} \mathrm{I}_{6}$ at $1.5 \mathrm{mM}$ in the syringe was injected into $280 \mu \mathrm{L} \mathrm{SPX} 1^{1-259 \Delta \mathrm{N} 17}$ at $70 \mu \mathrm{M}$ in the cell. To measure the binding affinity between SPX1 ${ }^{1-259}$ and PHR2 $230-426 \mathrm{~L} 348 \mathrm{~A} / \mathrm{L} 358 \mathrm{~A} / \mathrm{I} 362 \mathrm{~A}$ in presence or absence of $1 \mathrm{mM} \mathrm{Ins} \mathrm{P}_{6}, 40 \mu \mathrm{l} \mathrm{PHR} 2^{230-426 ~ \mathrm{~L} 348 \mathrm{~A} / \mathrm{L} 358 \mathrm{~A} / \mathrm{I} 362 \mathrm{~A}}$ at $120 \mu \mathrm{M}$ was injected into $280 \mu \mathrm{L}$ SPX1 $1^{1-259}$ at $10 \mu \mathrm{M}$. The ITC data were analyzed using Origin 7.0 and Microcal PEAQ-ITC analysis software.

Plant materials and growth conditions. The spx1 spx2 mutant was kindly offered from Dr. Chuanzao Mao (Zhejiang University, China). Hydroponic experiments were conducted using rice culture nutrient solution containing $500 \mu \mathrm{M} \mathrm{KH}_{2} \mathrm{PO}_{4}$. The nutrient solution was adjusted to $\mathrm{pH} 5.5$ by $\mathrm{KOH}$ and replaced every 3 days Experiments were carried out in a greenhouse with $16 \mathrm{~h}$ day $\left(30^{\circ} \mathrm{C}\right) / 8 \mathrm{~h}$ night $\left(22^{\circ} \mathrm{C}\right)$ photoperiod and photon density $200 \mu \mathrm{mol} \cdot \mathrm{m}^{-2} \bullet \mathrm{s}^{-1}$.

Generation of transgenic plants. The coding sequence of OsSPX1 was amplified and subcloned into pMD19-T vector. Mutations of InsP binding sites (Y25F/K29A K151A) in OsSPX1 were generated by PCR. The OsSPX1 and mutated OsSPX1 sequences were cloned into the pCAMBIA2301 vector. The $35 \mathrm{~S}$ promoters were replaced by the OSSPX1 native promoter. These two constructs were confirmed by sequencing and transformed into spx $s p x 2$ mutant through Agrobacteriummediated transformation. Sequences for primers are listed in Supplementary Table 3.

Quantification of Pi content. The Pi content of 3-week-old seedlings was measured using the phosphomolybdate colorimetric assay ${ }^{32}$. The shoots were collected after fresh weight measurement and ground into fine powder in liquid nitrogen. Inorganic phosphate $(\mathrm{Pi})$ was extracted in $1 \%$ acetic acid by repeated freezing and thawing twice. The supernatant was collected and then mixed with ammonium molybdate and ascorbic acid, in which $\mathrm{Pi}$ concentration was measured by colorimetric assay at $\mathrm{OD}=820 \mathrm{~nm}$.

Real-time quantitative PCR. Total RNA was extracted from root of 3-week-old rice plants using the RNeasy Plant Kit (Qiagen). $1 \mu \mathrm{g}$ total RNA was used for cDNA synthesis with PrimeScript RT reagent Kit (TaKaRa). Real-time qPCR was performed using a QuantiNova SYBR Green PCR Kit (Qiagen) on CFX96 real-time PCR detection system (Bio-Rad). Relative transcripts level were calculated by $2^{-\Delta \Delta \mathrm{Ct}}$ method according to the $\mathrm{Ct}$ values. Rice OsACT2 was used as the internal reference. Sequences for primers are listed in Supplementary Table 3.

Dual-LUC transient transcriptional activation assay. Protoplast isolation and transactivation assays are based on a previous report ${ }^{33}$. Hydroponic culture system was used for phrlphll mutant plants growth as described ${ }^{34}$. phrlphll mutant plants were grown in $1 / 2$ Hoagland nutrient solution for 4 weeks and transferred to Pi-depleted condition for $24 \mathrm{~h}$. About four-week-old plants were used for protoplast isolation. After transferring the plasmids, the protoplast from spx1spx2 mutant plants was incubated under Pi-replete condition for $12 \mathrm{~h}$. The harvested protoplasts were quantified with luciferase assay kit (Promega, USA), and LUC luminescence was measured with a plate reader (Perkin Elmer, USA). $\beta$ glucuronidase (GUS) reporter plasmid was used as internal control to normalize transfection efficiency in protoplast assays. Sequences for primers are listed in Supplementary Table 3.

Crosslinking experiment. Purified OsPHR2 $2^{230-426}$ protein was concentrated to $4 \mathrm{mg} \mathrm{ml}^{-1}$ in $25 \mathrm{mM}$ HEPES, pH7.0, $150 \mathrm{mM} \mathrm{NaCl}$. About $2 \mathrm{mg}$ of crosslinker DSS (disuccinimidyl suberate) was dissolved in $108 \mu \mathrm{l}$ DMSO to a final concentration of $50 \mathrm{mM} .2 \mu \mathrm{l}$ crosslinker DSS was added into $18 \mu \mathrm{l}$ protein sample at increasing concentration $(0,0.02,0.04,0.06,0.08$, and $0.10 \mathrm{mM}$,) according to the manufacturer's protocol. The reaction was carried out for $30 \mathrm{~min}$ at room temperature and stopped by the addition of $50 \mathrm{mM}$ Tris- $\mathrm{HCl}$ buffer, $\mathrm{pH}$ 8.0. After incubation of the mixture for $15 \mathrm{~min}$ at room temperature, the oligomeric state of samples was analyzed by SDS-PAGE electrophoresis. 
Thermal shift assay. Thermal shift assays were conducted with $15 \mu \mathrm{M}$ of SPX $1^{1-259}$ or SPX1 ${ }^{1-259 \Delta \mathrm{N} 17}$ in $20 \mathrm{mM}$ HEPES/NaOH pH 7.5, $200 \mathrm{mM} \mathrm{NaCl}$, and $5 \times$ dilution of SYPRO Orange dye (S6650, Thermo Fisher Scientific). The concentration of $\operatorname{InsP}_{6}$ ranges from 0 to $250 \mu \mathrm{M}$. Protein samples were heated with a $0.5^{\circ} \mathrm{C}$ per $10 \mathrm{~s}$ increasing gradient from 10 to $95^{\circ} \mathrm{C}$, the melt curve and melt temperature were recorded by the CFX Connect Real-Time PCR detection system (Bio-Rad).

Circular dichroism measurements. The $\mathrm{CD}$ spectra of the protein samples were recorded by a Chirascan ${ }^{\text {TN }}$-plus CD Spectrometer at the room temperature. The protein samples were concentrated to $25 \mu \mathrm{M}$ in $20 \mathrm{mM}$ HEPES/NaOH pH 7.5, $300 \mathrm{mM} \mathrm{NaF}$ in the absence or presence of $1 \mathrm{mM} \mathrm{Ins}_{6}$

SAXS data collection and analysis. The small angle X-ray scattering data were collected at the BL19U2 beamline at National Facility for Protein Science Shanghai (NCPSS) and Shanghai Synchrotron Radiation Facility (SSRF). $60 \mu \mathrm{L}$ of SPX1 $1^{1-259} 1.8 \mathrm{mg} / \mathrm{mL}$, InsP ${ }_{6}$-bound SPX $1^{1-259} 1.1 \mathrm{mg} / \mathrm{mL}$ were loaded in a quartz capillary. 2D scattering images were converted to 1D SAXS curves by BioXTAS RAW. The matching buffer scattering was subtracted from the sample scattering by PRIMUS. Pair distribution functions of the particles $\mathrm{P}(\mathrm{r})$ and the maximum sizes Dmax were calculated by the program GNOM. Low-resolution shapes were determined from solution scattering data using DAMMIF, from the ATSAS suite of programs. Twenty independent calculations were performed by DAMMIF programs for each data set, using default parameters and no symmetry constraints. Then twenty independent reconstructions were then averaged and filtered to a final consensus model using the DAMAVER suite. Rigid body modeling was performed using the program SASREF. We used SASREF to find relative positions of the helix $\alpha 1$ and the remaining part of SPX1 by inputting both models separately.

Reporting summary. Further information on research design is available in the Nature Research Reporting Summary linked to this article.

\section{Data availability}

Structural coordinates and structural factors have been deposited in the Protein Data Bank under accession number 7E40. Source data are provided with this paper.

Received: 10 April 2021; Accepted: 15 November 2021; Published online: 02 December 2021

\section{References}

1. Hughes, E. A. B., Robinson, T. E., Bassett, D. B., Cox, S. C. \& Grover, L. M. Critical and diverse roles of phosphates in human bone formation. J. Mater. Chem. B 7, 7460-7470 (2019).

2. Thomas, W. H. \& Dodson, A. N. Effects of phosphate concentration on cell division rates and yield of a tropical oceanic diatom. Biol. Bull. 134, 199-208 (1968).

3. Tapia-Torres, Y. et al. How to live with phosphorus scarcity in soil and sediment: lessons from bacteria. Appl. Environ. Microbiol. 82, 4652-4662 (2016).

4. Zohlen, A. \& Tyler, G. Soluble inorganic tissue phosphorus and calcicolecalcifuge behaviour of plants. Ann. Bot. 94, 427-432 (2004).

5. Wu, P., Shou, H., Xu, G. \& Lian, X. Improvement of phosphorus efficiency in rice on the basis of understanding phosphate signaling and homeostasis. Curr. Opin. Plant Biol. 16, 205-212 (2013).

6. Karthikeyan, A. S. et al. Phosphate starvation responses are mediated by sugar signaling in Arabidopsis. Planta 225, 907-918 (2007).

7. Yuan, H. \& Liu, D. Signaling components involved in plant responses to phosphate starvation. J. Integr. Plant Biol. 50, 849-859 (2008).

8. Walder, F. et al. Plant phosphorus acquisition in a common mycorrhizal network: regulation of phosphate transporter genes of the Phtl family in sorghum and flax. N. Phytol. 205, 1632-1645 (2015).

9. Nussaume, L. et al. Phosphate import in plants: focus on the PHT1 transporters. Front Plant Sci. 2, 83 (2011).

10. Sega, P. \& Pacak, A. Plant PHR transcription factors: put on a map. Genes https://doi.org/10.3390/genes10121018 (2019).

11. Zhou, J. et al. OsPHR2 is involved in phosphate-starvation signaling and excessive phosphate accumulation in shoots of plants. Plant Physiol. 146, 1673-1686 (2008).

12. Rubio, V. et al. A conserved MYB transcription factor involved in phosphate starvation signaling both in vascular plants and in unicellular algae. Genes Dev. 15, 2122-2133 (2001).

13. Secco, D. et al. The emerging importance of the SPX domain-containing proteins in phosphate homeostasis. N. Phytol. 193, 842-851 (2012)
14. $\mathrm{Hu}, \mathrm{B}$. \& Chu, C. Phosphate starvation signaling in rice. Plant Signal Behav. 6 927-929 (2011).

15. Wu, P. \& Xu, J. Does OsPHR2, central pi-signaling regulator, regulate some unknown factors crucial for plant growth? Plant Signal Behav. 5, 712-714 (2010).

16. Wu, P. \& Wang, X. Role of OsPHR2 on phosphorus homeostasis and root hairs development in rice (Oryza sativa L.). Plant Signal Behav. 3, 674-675 (2008).

17. Wang, Z. et al. Rice SPX1 and SPX2 inhibit phosphate starvation responses through interacting with PHR2 in a phosphate-dependent manner. Proc. Natl Acad. Sci. USA 111, 14953-14958 (2014).

18. Wild, R. et al. Control of eukaryotic phosphate homeostasis by inositol polyphosphate sensor domains. Science 352, 986-990 (2016).

19. Jung, J. Y., Ried, M. K., Hothorn, M. \& Poirier, Y. Control of plant phosphate homeostasis by inositol pyrophosphates and the SPX domain. Curr. Opin. Biotechnol. 49, 156-162 (2018).

20. Ried, M. K. et al. Inositol pyrophosphates promote the interaction of SPX domains with the coiled-coil motif of PHR transcription factors to regulate plant phosphate homeostasis. Nat. Commun. 12, 384 (2021).

21. Zhu, J. et al. Two bifunctional inositol pyrophosphate kinases/phosphatases control plant phosphate homeostasis. Elife https://doi.org/10.7554/eLife.43582 (2019).

22. Dong, J. et al. Inositol pyrophosphate InsP8 acts as an intracellular phosphate signal in arabidopsis. Mol. Plant 12, 1463-1473 (2019).

23. Jiang, M. et al. Structural basis for the target DNA recognition and binding by the MYB domain of phosphate starvation response 1. FEBS J. 286, 2809-2821 (2019).

24. Murase, K., Hirano, Y., Sun, T. P. \& Hakoshima, T. Gibberellin-induced DELLA recognition by the gibberellin receptor GID1. Nature 456, 459-463 (2008).

25. Yao, R. et al. DWARF14 is a non-canonical hormone receptor for strigolactone. Nature 536, 469-473 (2016).

26. Li, J. et al. The HAB1 PP2C is inhibited by ABA-dependent PYL10 interaction. Sci. Rep. 5, 10890 (2015).

27. Li, W. et al. Molecular basis for the selective and ABA-independent inhibition of PP2CA by PYL13. Cell Res. 23, 1369-1379 (2013).

28. Yin, P. et al. Structural insights into the mechanism of abscisic acid signaling by PYL proteins. Nat. Struct. Mol. Biol. 16, 1230-1236 (2009).

29. Kabsch, W. Xds. Acta Crystallogr. D. Biol. Crystallogr. 66, 125-132 (2010)

30. Adams, P. D. et al. PHENIX: a comprehensive Python-based system for macromolecular structure solution. Acta Crystallogr. D. Biol. Crystallogr. 66, 213-221 (2010)

31. Emsley, P., Lohkamp, B., Scott, W. G. \& Cowtan, K. Features and development of Coot. Acta Crystallogr. D. Biol. Crystallogr. 66, 486-501 (2010).

32. Jain, A. et al. Differential effects of sucrose and auxin on localized phosphate deficiency-induced modulation of different traits of root system architecture in Arabidopsis. Plant Physiol. 144, 232-247 (2007).

33. Fujii, $H$. et al. In vitro reconstitution of an abscisic acid signalling pathway. Nature 462, 660-664 (2009).

34. Zeng, H., Xia, C., Zhang, C. \& Chen, L.-Q. A simplified hydroponic culture of arabidopsis. Bio-Protoc. 8, e3121 (2018).

\section{Acknowledgements}

We thank Prof. Ning Zheng for critically reading our manuscript and for giving detailed comments and suggestions; the staff members of the BL17U1, BL19U1, and BL19U2 beamlines of National Facility for Protein Science in Shanghai (NFPS) at Shanghai Synchrotron Radiation Facility (SSRF) in China for support during crystallographic data and small angle X-ray scattering data collection; the staff members of the Large-scale Protein Preparation System at the National Facility for Protein Science in Shanghai (NFPS), Zhangjiang Lab, China for SEC-MALS data collection and analysis. M.L. is supported by Chinese Academy of Sciences and National Natural Science Foundation of China (31970580).

\section{Author contributions}

Experimental design: J.Z., Q.H., and W.X.; different constructs for protein purification, the complex assembling, crystallization, and crystal optimization: J.Z. and Q.H.; attempt in different species' protein for crystallization: H.L. and R.L.; structure determinations: D.Y.; structure analysis and various mutations design: J.Z., Q.H., and W.X; SEC assays: J.Z.; SEC-MALS and ITC assays: J.Z. and Q.H.; circular dichroism measurements: J.Z., J.Y., and C.W.; plant experiments design: J.Z., J.-K.Z., M.L., and W.X.; plant experiments: X.X. and S.G.; SAXS data collection and analysis: J.Z. and R.C.; thermal shift assay: J.Z. and Q.H.; manuscript writing: J.Z., Q.H., F.M., M.L., and W.X. with help from all of the co-authors.

\section{Competing interests}

The authors declare no competing interests. 


\section{Additional information}

Supplementary information The online version contains supplementary material available at https://doi.org/10.1038/s41467-021-27391-5.

Correspondence and requests for materials should be addressed to Mingguang Lei or Weiman Xing.

Peer review information Nature Communications thanks the anonymous reviewer(s) for their contribution to the peer review of this work. Peer reviewer reports are available.

Reprints and permission information is available at http://www.nature.com/reprints

Publisher's note Springer Nature remains neutral with regard to jurisdictional claims in published maps and institutional affiliations. (c) (P) Open Access This article is licensed under a Creative Commons Attribution 4.0 International License, which permits use, sharing, adaptation, distribution and reproduction in any medium or format, as long as you give appropriate credit to the original author(s) and the source, provide a link to the Creative Commons license, and indicate if changes were made. The images or other third party material in this article are included in the article's Creative Commons license, unless indicated otherwise in a credit line to the material. If material is not included in the article's Creative Commons license and your intended use is not permitted by statutory regulation or exceeds the permitted use, you will need to obtain permission directly from the copyright holder. To view a copy of this license, visit http://creativecommons.org/ licenses/by/4.0/.

(C) The Author(s) 2021 